\title{
Effects of visual restriction and unstable base dual-task training on balance and concentration ability in persons with stroke
}

\author{
Dong-Hoon Kim ${ }^{a}$, Kyung-Hun Kim ${ }^{b}$, Suk-Min Lee ${ }^{c}$ \\ ${ }^{a}$ Department of Physical Therapy, Bundang Jesaeng Hospital, Seongnam, Republic of Korea \\ ${ }^{b}$ Department of Physical Therapy, The Graduate School, Gimcheon University, Gimcheon, Republic of Korea \\ cDepartment of Physical Therapy, College of Health and Welfare, Sahmyook University, Seoul, Republic of Korea
} Objective: In the present study, the effects of visual restriction and unstable base dual-task training (VUDT), stable base dual-task
training (SDT), and on stroke patients' balance and concentration abilities were examined.

Design: Two-group pretest-posttest design.

Methods: Dual-task training was conducted for thirty persons with chronic stroke who were hospitalized or receiving physical therapy and were randomly assigned to either the VUDT group $(n=15)$ or the SDT group $(n=15)$. The subjects were divided into two groups of 15 participants each, the VUDT group and the SDT group. Dual-task training was administered for 30 minutes per session, three times a week for 8 weeks. The participants' balance was measured via the center of pressure migration distances, functional reach test (FRT), Berg Balance Scale (BBS), and attention was measured using the trail-making test and the Stroop test. Results: In comparisons within each group, the two groups showed significant differences before and after the training $(p<0.05)$. In the comparisons between the groups, the VUDT group showed significant improvements in center of pressure (COP), FRT, and BBS, and TMT compared to the SDT group $(p<0.05)$.

Conclusions: It would be more effective to conduct dual-task training as a rehabilitation training program under vision restriction and unstable supporting surface conditions than to conduct the test under unstable supporting plane conditions to improve balance and attention in chronic stroke patients.

Key Words: Dual task, Postural balance, Stroke

\section{Introduction}

Stroke is a disease in which circulatory disturbance in the blood vessels of the brain deprives the brain of oxygen, causing the necrosis of the brain cells [1] and neurological functional damage including sensory impairments, motor disturbance, speech disorders, visual impairment, perception disorders, and cognitive disorders. Stoke is one of the factors causing a decline in exercise ability among adults, and it is not curable for most patients [2]. Stroke patients have challenges in conducting high level tasks in sitting, standing, walking, and stair climbing [3], and they have an increased risk of falls due to increased sway and decreased walking ability [4].

A stroke patient with a decreased ability for recognition cannot actively participate in physical training for rehabilitation. Moreover, the patient transiently or permanently loses automatic postural control mechanisms, such as the ability to maintain a standing posture or the ability to perform dual tasks [5]. Due to the damage of the postural control mechanism, postural tasks are processed consciously and slowly rather than unconsciously and automatically; they are influenced by the higher centers of the cerebrum,

Received: 26 November, 2016 Revised: 18 December, 2016 Accepted: 19 December, 2016

Corresponding author: Suk-Min Lee

Department of Physical Therapy, College of Health and Welfare, Sahmyook University, 815 Hwarang-ro, Nowon-gu, Seoul 01795, Republic of Korea Tel: 82-2-3399-1632 Fax: 82-2-3399-1639 E-mail: leesm@syu.ac.kr

(c) This is an Open-Access article distributed under the terms of the Creative Commons Attribution Non-Commercial License (http://creativecommons.org/licens es/by-nc/4.0) which permits unrestricted non-commercial use, distribution, and reproduction in any medium, provided the original work is properly cited.

Copyright $@ 2016$ Korean Academy of Physical Therapy Rehabilitation Science 
which control attention [6].

Studies have been conducted on dual-task training to help rehabilitate the balance and concentration of stroke patients. According to task coordination and learning theory, single-task training has fewer processing requirements compared with dual-task training and does not allow the patient to perform two tasks at the same time. However, dual-task training allows a patient to conduct more than two tasks simultaneously, enabling coordination of various tasks [7]. In a study conducted by Hiyamizu et al. [8], the group that simultaneously performed recognition and movement tasks exhibited improved recognition task performance abilities, which showed that performing motor and recognition tasks at the same time affected one or both performances [9].

In Srivastava et al. [10] the effects of balance training by using visual feedback were evaluated and measured for improvement in complex balance and walking, as well as functional variables of chronic stroke patients with the use of a force plate. Kizony et al. [11] evaluated the walking ability of a single-task training group that performed only treadmill walking and an experimental group that conducted dual-task training using virtual reality; they found that while the single-task training group showed faster walking speeds than the experimental group, the experimental group showed greater differences in walking ability before and after the test. Furthermore, Bensoussan et al. [12] administered dual-task training to healthy participants and stroke patients where their vison was restricted to compare their balance ability; these researchers reported that balance ability was decreased in stroke patients.

In contrast to the types of research described above, few studies have been conducted on the balance and concentration abilities in persons with stroke with consideration of both visual restriction and base conditions related to a person's postural control ability. To increase the effects of dual-task training, visual effects [13] and base effects [14] should both be considered. In this regard, we conducted a study where persons with stroke performed dual-task training under visual restriction and unstable base conditions to examine changes in their balance and concentration ability and provide references that can help establish interventions to improve the balance and attention abilities in persons with stroke.

\section{Methods}

\section{Subjects}

Thirty patients receiving treatment in two rehabilitation hospitals in Korea were selected. The individuals had been diagnosed with stroke more than 6 months previously and did not have musculoskeletal system damage that could affect static standing balance, or aphasia affecting communication. Exclusion criteria were degenerative diseases and visual-somatosensory defect.

All participants had voluntarily agreed to participate in the study after they were informed of the study's objectives and procedures. In order to minimize selection bias, the subjects were assigned randomly and equally to one of two groups: visual restriction and unstable base dual-task training (VUDT, number 1), stable base dual-task training (SDT, number 2). Subjects received 8 weeks of dual-task training.

\section{Intervention}

The study was conducted from May 2016 to August 2016 with a total of 30 subjects who were divided into two groups of 15 each, the VUDT group and the SDT group. The training was conducted for 30 minutes per session, three times a week for eight weeks.

The VUDT group conducted two sets of training; standing on a stable surface with their eyes covered to remove visual information and were asked to maintain their posture while conducting recognition tasks; then, they were instructed to maintain balance on an unstable surface (balance pad) while conducting recognition tasks. The SDT group conducted two sets of 15-minute training where they maintained posture on a stable surface $(50 \mathrm{~cm} \times 50 \mathrm{~cm}$ in length and width), while conducting the above mentioned recognition tasks [8]. Recognition tasks were speaking random numbers, remember objects, completion of sentence, talking (Figure 1).

\section{Outcome measures}

Biorecue (analysis system by biofeedback, Marseille, France) was used to measure the center of pressure (COP). For measurement of COP moving distance, subjects were asked to maintain an upright position and keep their eyes forward while keeping their legs spread apart by 30 degrees for 1 minute. The functional reach test (FRT) was used to assess the subject's maximum reaching distance by having them stretch their arms out horizontally while maintaining their base of support in an upright and comfortable standing position. The FRT is known to be a simple and reliable testing tool that is used to assess stability with a reliability of $\mathrm{r}=0.98$ and a high inter-rater reliability of $\mathrm{r}=0.99$.

The Berg Balance Scale (BBS) is used to assess sitting, 


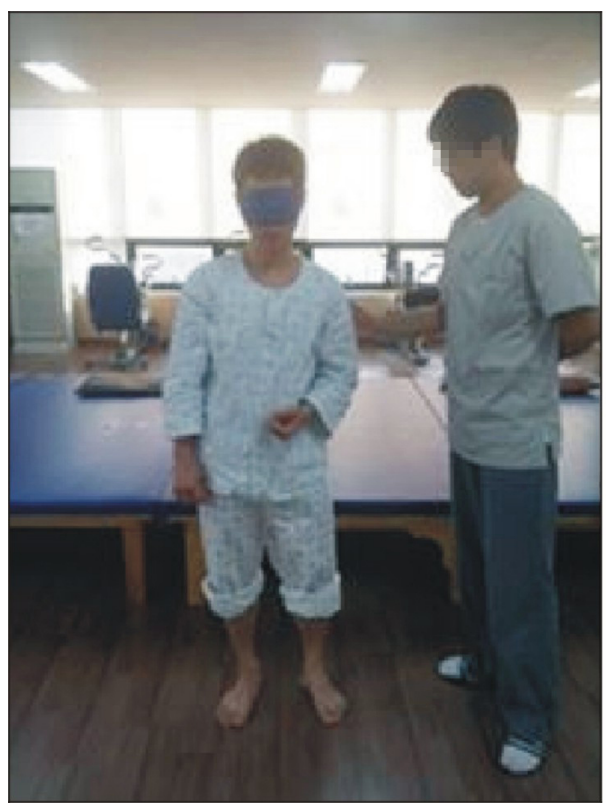

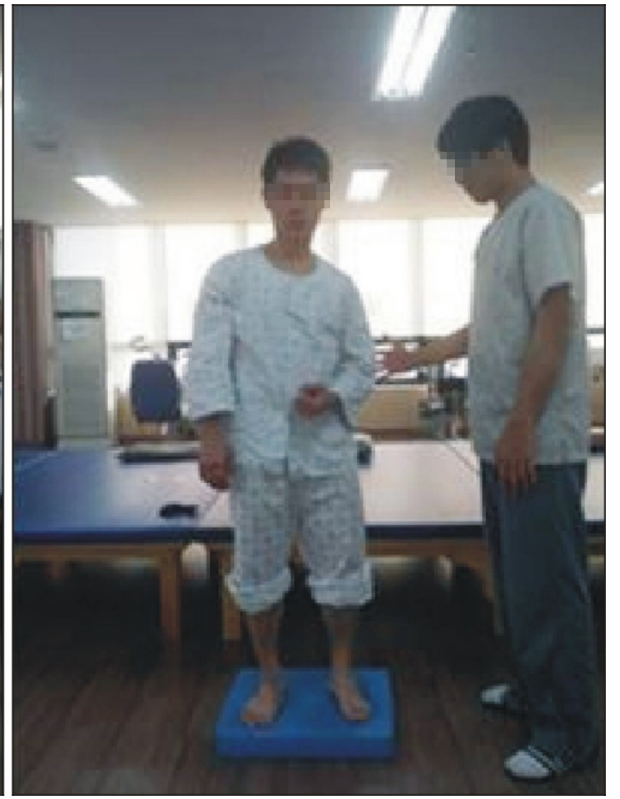

Figure 1. Visual restriction and unstable base dual-task training. standing, and postural change abilities. It has 14 items that can be scored on a range of $0(\min )$ to $4(\max )$ with a maximum possible score of 56 . All balance tests were performed three times and the average score was obtained. The BBS has a reliability of $\mathrm{r}=0.99$ and a high inter-rater reliability of $\mathrm{r}=0.98$.

The trail-making test (TMT) was conducted to test trails of complex concepts affecting the ability to cope with attention, recognition stage, and various stimulations. It has a reliability of $r=0.78$ and a high inter-rater reliability of $r=0.99$. In addition, the Stroop test was conducted to test selective attention. The evaluation items included total time to respond (Stroop test time) and the number of wrong answers (Stroop test error). All attention tests were conducted three times, and the mean values were recorded.

\section{Data and statistical analysis}

This study used the PASW Statistics ver. 18.0 program (IBM Co., Armonk, NY, USA). The chi-square test was conducted to verify general characteristics, such as gender, the paralyzed side, and types of stroke. The two-sample t-test was conducted to compare the homogeneity of dependent variables and differences between the two groups before and after the training. The paired t-test was conducted to compare differences before/after the training within the groups. To determine the effectiveness of the intervention, variation - the difference between mean values of dependent variables before/after the training - was used. The significance level for all statistical data was $\alpha=0.05$.

\section{Results}

The general characteristics of the study subjects are shown in Table 1. For balance, significant differences were observe in both groups before and after the training ( $p<$ $0.05)$. In a comparison of the two groups, the VUDT group showed significant improvement in the COP, the FRT, and the BBS scores compared to the SDT groups $(p<0.05)$. Regarding attention, significant differences $(p<0.05)$ were also shown in both groups before and after the training. In a comparison of the two groups, the VUDT group showed significant improvement in TMT compared to the SDT groups $(p<0.05$; Table 2).

\section{Discussion}

The COP moving distance decreased the most in the VUDT group. This result corresponded with the findings from previous studies where persons affected by stroke showed decreased sway after undergoing dual-task training $[13,15]$. Swan et al. [15] reported that postures with a high level of difficulty increase signals related to postural balance in various organs. It requires excessive attention to deal with the signals, creating exaggerated postural reactions in patients as they try to process them. However, conducting dual-task training appears to prevent tipping effects and decrease postural sway. Decreased COP moving distance during static standing represents decreased postural sway, which subsequently indicates increased stability during stat- 
Table 1. The general characteristics of the subjects $\quad(\mathrm{N}=30)$

\begin{tabular}{lrrr}
\hline & VUDT $(\mathrm{n}=15)$ & SDT $(\mathrm{n}=15)$ & \multicolumn{1}{c}{$\mathrm{t}(p)$ or $\chi^{2}$} \\
\hline Age $(\mathrm{y})$ & $58.07(11.28)$ & $52.00(9.20)$ & $1.615(0.118)$ \\
Heigth $(\mathrm{cm})$ & $161.17(6.21)$ & $161.25(7.52)$ & $-0.034(0.973)$ \\
Weight $(\mathrm{kg})$ & $61.03(6.81)$ & $61.82(6.52)$ & $-0.326(0.747)$ \\
MMSE & $28.00(1.13)$ & $27.33(1.35)$ & $1.468(0.153)$ \\
Gender & & & \\
$\quad$ Male & $5(33.3)$ & $8(53.3)$ & $1.222(0.269)$ \\
Female & $10(66.7)$ & $7(46.7)$ & \\
Diagnosis & & & \\
$\quad$ Infarction & $11(73.3)$ & $7(46.7)$ & $2.222(0.136)$ \\
Hemorrhage & $4(26.7)$ & $8(53.3)$ & \\
Affected side & & & \\
$\quad$ Left & $9(60.0)$ & $7(46.7)$ & $0.136(0.713)$ \\
Right & $6(40.0)$ & $8(53.3)$ & \\
\hline
\end{tabular}

Values are presented as mean (SD) or $\mathrm{n}(\%)$.

VUDT: visual restriction and unstable base dual-task training, SDT: stable base dual-task training, MMSE: mini mental state examination.

ic standing.

Regarding the FRT, the VUDT group showed greater improvement than the SDT group. This result was similar to that of a study conducted by Smania et al. [16] that showed that persons with chronic stroke showed significant changes in balance and gait speeds when comparing before and after balance training on an unstable surface. The result was also similar to that of a study conducted by Bonan et al. [17], which showed that persons with chronic stroke showed significantly improved balance after performing vision-restricted training. Granacher et al. [18] reported that balance training conducted on an unstable surface sensitizes muscle spindles through gamma motor neurons; this improves motor output, which in turn influences the stiffness of joints. Since the FRT measurements are dependent on both lower body stability and upper body flexibility, improved FRT scores may indicate improved ability to perform daily life activities.

For the BBS, the VUDT group showed greater improvement than the SDT group. The result was similar to that of Her et al. [13], which showed significant improvements in the Berg balance test after training in the motor dual-task group, the recognition dual-task group, and the motor and recognition dual-task group. The result corresponded with that of Maxwell and Master [19], which showed that facilitating external focus, concentrating on external factors such as an object in front of a chair or the sway of water in a cup, facilities postural control more than an internal focus, where
Table 2. Comparison of balance and attention ability between

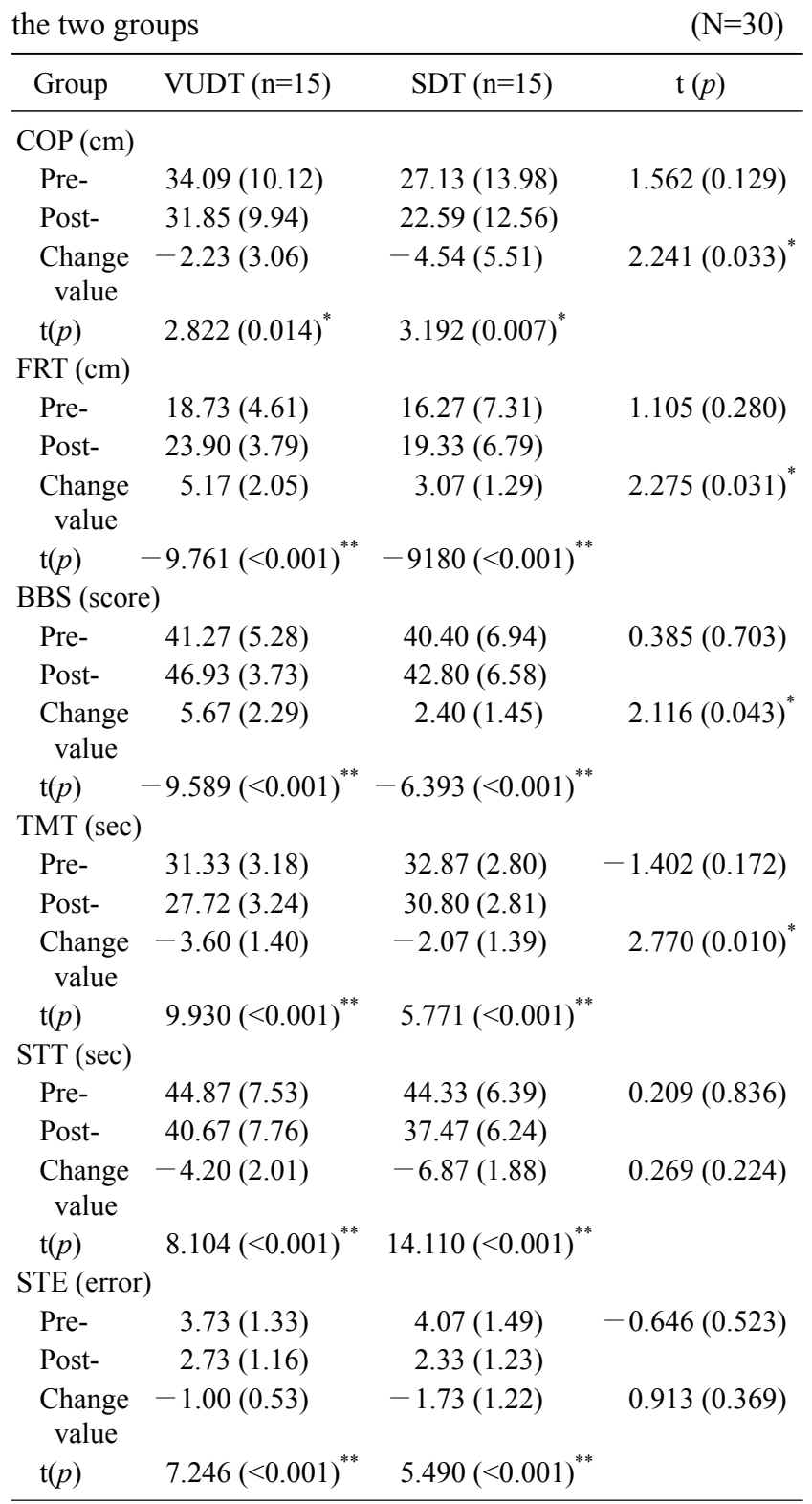

Values are presented as mean (SD).

VUDT: visual restriction and unstable base dual-task training, SDT: stable base dual-task training, COP: center of pressure, FRT: functional reach test, BBS; Berg balance scale, TMT: trail-making test, STT: Stroop test time, STE: Stroop test error. ${ }^{*} p<0.05, \stackrel{* *}{p}<0.01$.

subjects focus on their movement. Improved BBS scores indicate improved balance in persons with stroke since it measures balance ability while performing various tasks, thereby providing high reliability and internal validity.

For the TMT and Stroop test evaluating attention, the VUDT group showed greater improvements compared to the SDT group. The TMT tested the dichotomy of attention, 
recognition stage, and trails of complex concepts, while the Stroop test tested selective attention. Improvements in the test results can be interpreted to show that attention has improved in stoke patients. This result corresponded with the findings of Hiyamizu et al. [8]. The VUDT group used various balance conditions that included rotational and divided attention as an intervention for a person affected by stroke with loss of attention, as described by Michel and Mateer [20]. It is implies that intensive task training improved attention.

This study has some limitations in that it generalized test results for all stroke patients. In addition, since we could not completely control the daily lives of the subjects, we could not totally exclude the possibility that other factors may have affected the balance and attention of the subjects. Another limitation is that only a recognition dual task was used among the possible dual tasks as an experimental method. In this regard, further studies are needed on various dual-task training methods, differing results due to levels of difficulty, and the inclusion of brainwave tests when evaluating attention-related items.

In this study, we confirmed that dual-task training with vision restriction and unstable surface produced a positive effect on balance and attention abilities in person with chronic stroke. Therefore, it would be more effective to conduct dual-task training as a rehabilitation training program with the inclusions of vision restriction combined with unstable surface conditions rather than to conduct a study with only unstable support conditions in order to improve balance and attention abilities in persons with chronic stroke.

\section{Conflict of Interest}

The authors declared no potential conflicts of interest with respect to the authorship and/or publication of this article.

\section{References}

1. Kolb B, Gibb R. Brain plasticity and recovery from early cortical injury. Dev Psychobiol 2007;49:107-18.

2. Cramer SC, Bastings EP. Mapping clinically relevant plasticity after stroke. Neuropharmacology 2000;39:842-51.

3. Brown LA, Sleik RJ, Winder TR. Attentional demands for static postural control after stroke. Arch Phys Med Rehabil 2002;83: 1732-5.

4. Belgen B, Beninato M, Sullivan PE, Narielwalla K. The association of balance capacity and falls self-efficacy with history of falling in community-dwelling people with chronic stroke. Arch
Phys Med Rehabil 2006;87:554-61.

5. Huxhold O, Li SC, Schmiedek F, Lindenberger U. Dual-tasking postural control: aging and the effects of cognitive demand in conjunction with focus of attention. Brain Res Bull 2006;69:294305 .

6. Hyndman D, Ashburn A. People with stroke living in the community: Attention deficits, balance, ADL ability and falls. Disabil Rehabil 2003;25:817-22.

7. Silsupadol P, Siu KC, Shumway-Cook A, Woollacott MH. Training of balance under single- and dual-task conditions in older adults with balance impairment. Phys Ther 2006;86:269-81.

8. Hiyamizu M, Morioka S, Shomoto K, Shimada T. Effects of dual task balance training on dual task performance in elderly people: a randomized controlled trial. Clin Rehabil 2012;26:58-67.

9. Bherer L, Kramer AF, Peterson MS, Colcombe S, Erickson K, Becic E. Training effects on dual-task performance: are there age-related differences in plasticity of attentional control? Psychol Aging 2005;20:695-709.

10. Srivastava A, Taly AB, Gupta A, Kumar S, Murali T. Post-stroke balance training: role of force platform with visual feedback technique. J Neurol Sci 2009;287:89-93.

11. Kizony R, Levin MF, Hughey L, Perez C, Fung J. Cognitive load and dual-task performance during locomotion poststroke: a feasibility study using a functional virtual environment. Phys Ther 2010;90:252-60.

12. Bensoussan L, Viton JM, Schieppati M, Collado H, Milhe de Bovis $\mathrm{V}$, Mesure $\mathrm{S}$, et al. Changes in postural control in hemiplegic patients after stroke performing a dual task. Arch Phys Med Rehabil 2007;88:1009-15.

13. Her JG, Park KD, Yang YA, Ko T, Kim H, Lee J, et al. Effect of balance training with various dual-task conditions on stroke patients. J Phys Ther Sci 2011;23:713-7.

14. Hyndman D, Pickering RM, Ashburn A. Reduced sway during dual task balance performance among people with stroke at 6 and 12 months after discharge from hospital. Neurorehabil Neural Repair 2009;23:847-54.

15. Swan L, Otani H, Loubert PV, Sheffert SM, Dunbar GL. Improving balance by performing a secondary cognitive task. $\mathrm{Br}$ J Psychol 2004;95:31-40.

16. Smania N, Picelli A, Gandolfi M, Fiaschi A, Tinazzi M. Rehabilitation of sensorimotor integration deficits in balance impairment of patients with stroke hemiparesis: a before/after pilot study. Neurol Sci 2008;29:313-9.

17. Bonan IV, Yelnik AP, Colle FM, Michaud C, Normand E, Panigot B, et al. Reliance on visual information after stroke. Part II: Effectiveness of a balance rehabilitation program with visual cue deprivation after stroke: a randomized controlled trial. Arch Phys Med Rehabil 2004;85:274-8.

18. Granacher U, Gollhofer A, Strass D. Training induced adaptations in characteristics of postural reflexes in elderly men. Gait Posture 2006;24:459-66.

19. Maxwell JP, Master RSW. External versus Internal Focus Instructions: Is the learner paying attention? Int J Appl Sport Sci 2002;14:70-88.

20. Michel JA, Mateer CA. Attention rehabilitation following stroke and traumatic brain injury. A review. Eura Medicophys 2006;42: 59-67. 